Original Contribution

\title{
SUITABILITY OF THE MODALITY VIRTUAL BRONCHOSCOPY WITH ASPIRATION OF A FOREIGN BODY
}

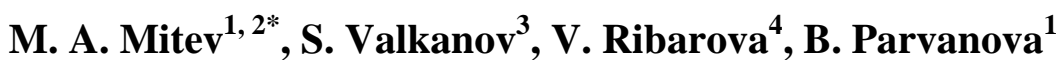 \\ ${ }^{1}$ Department of Medical Physics, Biophysics, Roentgenology and Radiology, Trakia University, \\ Stara Zagora, Bulgaria, \\ ${ }^{2}$ Department of Diagnostic Imaging, University Hospital “St. Kirkovich”, Trakia University, \\ Stara Zagora, Bulgaria \\ ${ }^{3}$ Department of Surgery, Neurosurgery and Urology and Anesthesiology, Clinic of Neurosurgery, \\ Trakia University, Stara Zagora, Bulgaria \\ ${ }^{4}$ Department of Diagnostic Imaging, General Hospital - Sliven, Bulgaria
}

\begin{abstract}
Aspirations of foreign bodies are life-threatening among children and elderly patients requiring urgent medical assistance. The aim of the study is to present summarized results from various authors' studies to reveal VB's diagnostic abilities for suspected aspiration of a foreign body. VB has been shown to be a particularly useful non-invasive modality for the complex tracheobronchial tree assessment for suspected aspiration of a foreign body due to its high sensitivity, specificity and validity. MDCT VB with MPR allows accurate localization of the foreign body, but in secondary inflammatory changes and secretions it does not provide accurate information about the form and type of the finding. VB cannot replace FB ("gold standard") but successfully supports and complements it. MDCT could provide early diagnosis in cases of suspected aspiration of a foreign body in children and adults and avoid real bronchoscopy in patients with poor overall condition.
\end{abstract}

Key words: Foreign body, Multidetector computed tomography, Virtual bronchoscopy

\section{INTRODUCTION}

Virtual Bronchoscopy with Multidetector Computer Tomography (VB MDCT) is a noninvasive technique providing an internal view of the trachea and large bronchi through threedimensional reconstruction $(1,2)$. Aspiration of a foreign body is common in children under the age of 3, for whom MDCT and VB lowdose modalities are particularly suitable. Foreign bodies are with high risk as a cause of death among children, rarely in adults, and require urgent attention from the medical staff (3-9; etc.). Negative VB inspections with MDCT exclude classical invasive

\footnotetext{
*Correspondence to: Mitko Mitev, Department of Medical Physics, Biophysics, Roentgenology and Radiology, Department of Diagnostic Imaging, Faculty of Medicine, Department of Diagnostic Imaging, University Hospital "St. Kirkovich”, Trakia University - Stara Zagora, 6000 Stara Zagora: +35988770 6079, E-mail: mitev.mitko69@gmail.com
}

bronchoscopy $(3,4)$. CT VB has been shown to be particularly useful in foreign body aspiration screening because of its high sensitivity, specificity and validity (2). The study aims to present summarized results from various authors' studies to reveal VB's diagnostic abilities for suspected aspiration of a foreign body.

\section{RESULTS AND DISCUSSION}

The first data on clinical use of bronchoscopy dates from the end of the 19th and the beginning of the 20th century, incl. for patients in infancy (10). Bronchoscopy has established itself over the years as being commonly used to diagnose and treat a wide range of lung diseases and as a major tool in medicine in critical surgery, thoracic surgery, rhinolaryngology and pediatric pulmonology. At the present stage it is defined as the most commonly used minimally invasive diagnostic procedure for pulmonary diseases (11). 
The first successful extraction of a foreign body (a bone from the right bronchus) was made by Gustav Killian in 1897 to a 63-yearold man by an esophagoscope. Then rigid bronchoscopy (RB) becomes a preferred procedure for removing foreign bodies from the respiratory tract until the appearance of the flexible bronchoscopy (FB) in the early 1970s. FB expands the scope of bronchoscopic interventions to the peripheral airways, allowing the removal of foreign bodies located more distantly with a wide variety of special flexible tools. Fiberoptic bronchoscopy, however, remains the traditional gold standard (12).

$\mathrm{RB}$ and FB are perceived as appropriate modalities widely applied to suspected aspiration of a foreign body. They are a preferred tool for diagnosing foreign bodies in adult patients. They are also used to remove foreign bodies without additional risk for patients against more invasive procedures, e.g. surgical intervention $(13,14,7,15,16,12,17$, $18,19,6,20,8,9$, etc.). In some cases, however, foreign bodies may imitate other disorders that delay diagnostics or certain factors may require the application of other modern modalities such as MDCT VB. It is known that if the foreign body falls into the larynx area, the body reacts immediately to the effects of asphyxiation, but if the foreign body penetrates down into the bronchial lumen, the sensation can be even without symptoms, making it difficult to diagnose without bronchoscopy. X-rays are often considered as unreliable in the aspiration of X-rays negative foreign bodies, as opposed to MDCT VB, allowing objective assessment of lung, bronchi and mediastinum by non-invasive diagnosis and final intervention planning (21). The multimodal approach to diagnosis has always been considered useful (22, etc.). The application of radiographic methods and their accuracy is defined as non-specific for foreign body aspiration diagnostics (23, etc.). Studies on the diagnostic capabilities of MDCT VB for suspected aspiration of a foreign body are extremely few. Aspirations of a foreign body have been reported mainly for children under the age of $3(1,24,5,3,25,21,26,27,28,29$, 6 , 4, etc.) but although significantly less common, they are categorized as lifethreatening for elderly patients too (30-6, etc.).

Haliloglu et al (1) presented the results of CT $\mathrm{VB}$ in assessing children with suspected aspiration of a foreign body. They studied 23 children (11 boys and 12 girls) with an average age of 2.4 years ( 8 months - 14 years). They performed chest radiograms, spiral CT scan, FB and VB images. CT VB and FB revealed the location of a foreign body in 7 patients (to the left of the right bronchus in 4 patients, in the lower right bronchus lobe in one patient and to the left to the main bronchi in 2 patients). There were no inconsistencies between the two modalities. Koşucu et al (24) investigated the potential of the low-frequency MDCT VB for assessing children with suspected foreign body aspiration. They studied 23 patients (10 girls, 13 boys) of average age 3.3 years (from 9 months to 13 years). At an average flow of the tube $35 \mathrm{~mA}$ (range, 25-50mA), they obtained excellent image quality for 9 children (39\%), good image quality for 12 children (52\%) and poor image quality for 2 children (9\%). Artefacts were found in 5 children. In 15 patients, foreign bodies were established by both methods. They concluded that the assessment of aspiration of foreign bodies of the respiratory tract in children could be done by using a low-flow MDCT protocol. Kocaoglu et al (5) explored the possibilities of VB in children with suspicions of inhalation of foreign bodies. The authors performed a thin profile axial MDCT and multiplanar preformatted imaging (MPR). They determined the importance of MDCT VB with MPR compared to conventional bronchoscopy in assessing children with suspected foreign body aspiration. They studied 21 children (14 boys, 7 girls, 8 months to 7 years old, mean age 3.5 years). Studies were performed with 16-layer MDCT using 100-120kV, 30-50mA, $1 \mathrm{~mm}$ profile thickness, 1.2 pitch and 0.6$1.0 \mathrm{~mm}$ reconstruction interval. Prior to $\mathrm{CT}$, for all cases they received also chest radiographies. After MDCT studies, they performed VB and MPR imaging. They identified 9 foreign bodies using standard conventional bronchoscopy. Through VB, thinprofile axial MDCT and MPR they identified 8 of 9 foreign bodies. CT with MPR images and 
VB did not show, according to the authors, a chronic foreign body. In one patient, the endobronchial mucosa was diagnosed as a foreign body. Adaletli et al (3) published the results of the use of low-dose multidetector CT and VB in children with suspected foreign body aspiration. They explored potential uses of low-dose MDCT and VB for assessing and manipulating the suspicions of aspiration of a foreign body in 37 children (17 girls, 20 boys, aged from 4 months to 10 years, average age 32 months). Before MDCT they performed chest radiographies on all patients. They applied MDCT using low-dose equipment. In the same session, they obtained VB images. Conventional bronchoscopy was performed within 24 hours for patients with obstructive abnormalities through MDCT and VB. In 13 patients the foreign bodies were detected and removed by FB. They reported localization of the foreign bodies to the right of the main bronchi $(\mathrm{n}=5)$, the middle of the bronchi $(\mathrm{n}=$ 6 ), the medial segment of the middle bronchus lobe $(\mathrm{n}=1)$ and to the left of the main bronchi $(n=1)$. In the remaining 3 patients, they received a false-positive diagnosis of obstructive pathology with MDCT and VB. They gave definitive diagnosis of secretions ( $\mathrm{n}$ $=2)$ and schwannoma $(n=1)$ proved by FB. In 21 patients without obstructive pathology after MDCT and VB they did not perform FB. Cevizci et al (25) carried out VB studies as a dynamic modality for diagnosis and manipulation of suspected aspiration of a foreign body. They pointed out that rigid bronchoscopy (RB) was an invasive procedure and had its own risks. They evaluated the place of VB in manipulating patients with clinical and radiological suspicions of aspiration of a foreign body to determine when RB can be avoided in their use. They studied 60 patients ( 35 boys and 25 girls) aged between 4 months and 7 years with clinical and x-ray suspicions of aspiration of a foreign body. Patients with aspiration of radio negative foreign bodies were excluded from the study and treated directly with RB. They performed VB using 16 MDCTs. If the clinical study and the RB maintained the diagnosis of a foreign body, they performed the RB for diagnosis and manipulation. The results from RB they compared with those from VB. Bhat et al. (2)
MITEV M. A., et al. compared $\mathrm{VB}$ and $\mathrm{FB}$ in children with tracheobronchial foreign bodies not detected by conventional X-ray. Ordinary radiography they initially performed in 40 children with suspicion of aspiration of a foreign body. They performed CT VB in the 20 children with chest radiographies seeming normal. They made RB to all patients. They then compared the virtual bronchoscopic findings with the RB results. In 12 patients, foreign bodies detected by VB, were confirmed by RB. In one case, the mucosal canal was perceived as a foreign body by VB. In another case, a little foreign body is omitted in VB. Kostadinov (37) points out those foreign bodies in the trachea and bronchi are an urgent condition requiring immediate attention, especially in children under 6 years of age and very rarely in adults. The use of low doses of MDCT and VB, according to the author, may help to detect a foreign body that is not detected by survey radiography. If CT shows, in his view, signs of aspiration of a foreign body, the patient must be subjected to an endoscopic examination for final diagnosis and treatment. Sattar et al (23) investigated the diagnostic accuracy of X-ray in tracheobronchial aspiration of foreign bodies in children. They performed multiplanar image processing after MDCT examination. The difference between MDCT and conventional X-Ray they defined as being statistically significant ( $p<0.001$ ). The sensitivity, specificity, positive predictive value (PPV), negative predictive value (NPV) and chest Xray accuracy obtained by them are: $66.6 \%$, $50 \%, 89.6 \%, 18.7 \%$ 4\%. Wani et al (21) indicated that the aspiration of a foreign body in children under the age of 3 might manifest acute respiratory difficulties, suffocation and sharp wheezing, or might be asymptomatic. Sensitivity to the foreign body was due to the small caliber of the airways, incomplete laryngeal closure, and inadequate swallowing reflexes at this age. Bronchial occlusion could lead to pneumonia, pulmonary abscess, bronchiectasis, and rare pneumomediastinum. The authors described a case of a child with a bronchial aspiration of a foreign body, manifested by subcutaneous emphysema and pneumomediastinum. Jung et al (26) identified the bronchial foreign bodies in children as difficult to diagnose when there was no 
witness of the aspiration event. They applied 3D VB (3D-CTVB) to diagnose bronchial foreign bodies. They studied 10 children (M: F $=5:$ 5). They analysed the physical, radiological and bronchoscopic findings of the patients. Four patients (44\%) witnessed the aspiration event. In nine patients $(90 \%)$ on a conventional X-ray machine they reported hypoventilation and hypoaerisation of the affected lung. In all patients, the location of a foreign body in preoperative studies by 3DCTVB corresponded to the intraoperative bronchoscopic findings. All foreign bodies were successfully removed by rigid bronchoscopy. Boninska and Borisova (38) presented imaging methods for diagnosing a foreign body in the respiratory system. The authors emphasized that using low doses of MDCT could help to detect a foreign body that was not seen in the survey radiography, and if CT showed signs of aspiration of a foreign body, the patient should undergo an endoscopic examination for final diagnosis and treatment. They concluded that the imaging diagnosis was an integral part of modern medicine. Hou et al (30) reviewed 617 cases of inhalation and swallowing of foreign bodies in dental procedures and the importance of comprehensive documentation to them for the purposes of treatment and prevention. Richards (27) applied VB (CT) as a non-invasive alternative for diagnosis and localization of aspirated foreign bodies in children under the age of 4 , with a peak of incidents between the first and second year. Srivastava and Tyagi (28) investigated the utility of VB in assessing suspected aspiration of a foreign body in the tracheobronchial tree. They performed a retrospective and prospective study of 100 patients with a history of sudden breathlessness, sudden coughing, with or without cyanosis and with or without fever. They found the highest encounter of foreign bodies, i.e. $61 \%$ in children aged $1-5$ (73\% in boys and $27 \%$ in girls). Frequency of encounter of a foreign body, according to the authors, was much higher in the low socioeconomic status group (63\%) compared to the average status (34\%). As foreign bodies they found: grains of Indian pepper (31\%), fruits of the tropical Annona tree (Annona squamosa sugar apple, 22\%), chickpeas (8\%), tamarind
MITEV M. A., et al. seed (tropical tree Tamarindus indica), (6\%), coconut $(5 \%)$ and peanut $(10 \%)$. In these studies they reported lumen on the right main bronchus as the prevalent localization of the foreign body (57\%), followed by the upper trachea (22\%) and the left bronchus lumen $(21 \%)$. Yajima et al (31) examined the case of aspiration of a sewing pin by an 18-year-old girl. Due to the complications known in the literature in removing sharp objects such as pins, the authors approached with great care to avoid injury to trachea and vocal cords. By CT VB they diagnosed and then, to prevent injury to the trachea and the vocal cords, apply FB with a truncated endobronchial tube. According to Yogi et al (29), because most foreign bodies are not X-rays negative, about one-third of children had normal radiographies. They investigated the diagnostic accuracy of VB vs. RB in assessing children with suspected foreign bodies. They studied 37 patients aged 12 and less than 12 years who were admitted in hospital for a period of 1,5 years and passed VB. They detected foreign bodies in children mostly aged 1-3 years and predominantly of male gender. Foreign body was found in $64.86 \%$ of cases. By normal radiography, they reported a foreign body in $27.02 \%$ of the cases and by RB in $75.67 \%$. They indicated the peanuts as the most commonly aspirated foreign bodies. Elhamady et al (32) conclude that foreign bodies in the respiratory - digestive tract are usually observed in children or in adults. They analysed the frequency, the risk factors, the location, and the clinical manifestations, the different ways of extracting tracheobronchial and oesophageal foreign bodies in patients with aspiration or swallowing of a foreign body. They presented 90 patients with presumed foreign body aspiration and 100 patients with presumed foreign body swallowing. They performed X-ray, computer scan, ultrasound and VB. Some patients underwent rigid esophagoscopy / bronchoscopy in general anaesthesia based on clinical and radiological data. Other patients underwent surgical interventions. They found that for the aspiration of a foreign body there was statistically significant gender difference among teenagers and adults; women were more likely to be affected than men. Aspiration 
of a foreign body mainly occurred in teenagers (> 10-20 years) and adults (> 20 years). A foreign body in the right main bronchus was more likely to be found in children less than 10 years of age. Rigid bronchoscopic extraction of foreign bodies was the most common extraction method observed in 70 patients (77.8\%). They found that coins were the most swallowed foreign body; the upper esophageal sphincter (UES) was the most common place of foreign body retention. Foreign bodies in the respiratory-digestive tract they defined as a constant danger in all age groups, especially in children and elderly people, requiring immediate action and extraction. Hosseinpour and Kolahdouzan (6) emphasized that aspiration of a needle was a rare condition. Sometimes there were some difficulties in diagnosing and managing this type of foreign body, especially in children. They presented a case of a 9-year-old boy with aspiration of a needle. They indicated that despite significant advances in airway management and endoscopic technology, foreign respiratory bodies still leaded to significant morbidity and were an important cause of death in children. James et al (4) indicated that foreign bodies were with high risk of causing death among children and required urgent attention from medical staff. While most cases were easily diagnosed with a clinical history of acute respiratory distress, some cases remained less painful and were manifested later. They reported a case with a 7-year-old boy who aspired a LEGO toy and presented a weekly increase in asthma-compatible respiratory stress. Despite the normal X-ray, low-dose CT indicated the presence of a foreign body in the left main bronchus, which was subsequently removed by FB. Mitev $(34,35)$, Mitev et al (36) evaluated the relevance of VB with MDCT in patients (children and adults) with suspected foreign bodies. They indicated VB's major role in preliminary evaluation of the tracheobronchial tree and as a source of valuable information for subsequent real bronchoscopy. Through VB they achieved full compliance in the localization of foreign bodies. Regarding the shape and appearance of foreign bodies, as well as changes in the mucosa, they found that the VB method had no high sensitivity. VB MDCT can evaluate
MITEV M. A., et al. bronchi distally and in the neighbourhood and because it is non-invasive it can be successfully used in children and adult patients with impaired general condition. As a result of axial and multiplanar imagery, the assessment is complex, accurate and correct. VB is a very useful modality in the aspiration of foreign bodies, but it cannot replace FB, especially in case of old processes, with secondary changes and secretions that do not allow a clear definition of the form and type of the pathological finding in VB. Pitiot et al (39) indicated that aspiration of a foreign body was a risky situation common to children. As a "gold standard" for diagnosis and removal, they indicated RB requiring general anesthesia and leading to possible complications in 4-17\% of cases. Advances in radiology allow CT scanners to quickly obtain results with high diagnostic value that could be used as an alternative to RB. They provided a retrospective analysis of 200 children (average age 30 months) with suspicions of aspiration of a foreign body. Of these, a foreign body was found in 59 cases and in 9 cases false positive results were reported. One child was examined three months later for asthma without reporting a second suffocation. A new CT scan found a foreign body removed via VB. This was the only false negative result. The obtained negative estimated value of CT was $99.2 \%$ and the positive estimated value was $83.8 \%$. The use of CT-scan with multi-planar reconstruction for supposed aspiration of a foreign body is a reliable alternative to endoscopy under general anesthesia, especially in asymptomatic patients, avoiding too many negative endoscopies. Reynisson et al (40) emphasized that VB did not meet the need for pulmonologists to detect, define and sample common targets outside the bronchial wall. Therefore, they developed and explored a new visualization technique for navigational bronchoscopy. They investigated it on nonselective CT data from eight patients. They found that attached to a centred curved surface (ACCuSurf), it facilitates the detection and interpretation of anatomical signs. Semple et al (41) indicated that "major airway disease" was the phrase encompassing a wide variety of pathological conditions affecting the trachea, the main, lobar, segmental, and proximal 
MITEV M. A., et al.

subsegmental bronchi. They divided the corresponding pathologies into focal or diffuse, as well as states with classical manifestations in computed tomography. Although, the spatial and temporal resolution of modern multi-point CTs is well adapted to the representation of small structures such as peripheral airways, concerns about exposure to radiation and growing interest in the role of functional and quantitative imaging have led to a leap in dose reduction studies in $\mathrm{CT}$ and structural and functional imaging of the airways by magnetic resonance imaging. Tartar et. al. (42) investigated the volume changes in the diagnosed materials to determine the optimum removal time for each type of foreign body (peeled and blanched peanuts, roasted chickpeas, hazelnuts, walnuts, almonds, pistachios, watermelons, unmilled sunflower seeds, pumpkin seeds without husk, pumpkin seeds, beans, lemon seeds and apple seeds) with VB application. Tsang et al (8) reported a 7-year-old boy with an aspired toy "LEGO" and a weekly history of increasing respiratory fear compatible with some asthma. Despite the normal chest X-ray analysis, low-dose CT indicated the presence of a foreign body in the left bronchus, which was subsequently removed through FB.

Foreign air bodies are the leading cause of death among children and rarely in adults and require urgent assistance. Cases are easily diagnosed through the clinical history of acute respiratory distress. Some cases remain less painful and appear later (8). VB is a newly introduced research technique complementing FB in tracheobronchial tree assessment as well as a promising method for the diagnosis of lung diseases $(43,44)$. When chest X-ray is normal, and clinical diagnosis suggests aspiration of a foreign body, research suggests that CT VB should be applied and RB should be avoided (1). The exact determination of the location of the foreign body before bronchoscopy and of a foreign body in patients with little suspicions and normal or nonspecific findings on thoracic radiography was achieved with low-flow MDCT VB Koşucu et al (24). Kocaoglu et al (5) emphasized that MDCT with VB and axial / MPR images provided equally valuable information in children with suspected aspiration of a foreign body and that VB prevented unnecessary conventional bronchoscopic research. However, according to the authors, VB increased the duration and cost of the examination by not providing additional information through MPR imaging when assessing a foreign body aspiration. According to Adaletli et al (3), low-dose MDCT and VB are non-invasive radiological modalities that could be easily used in the study of suspected foreign body aspiration in children. MDCT and VB provide the exact location of obstructive pathology before FB. The authors emphasize that if the obstructive pathology is depicted with MDCT and VB, FB is performed either to confirm the diagnosis or to diagnose an alternative cause of obstruction. They point out that when obstructive pathologies are not detected by MDCT and VB, FB could not be clinically useful. The positive VB, according to Cevizci et al (25), helps to reduce the operative time by providing information on the localization and the size of the foreign body. The low-dose MDCT with VB, according to Wani et al (21), enhances detection and helps in localizing the intraluminal foreign body and thus speeds up proper management. Removal of the foreign body is performed with a rigid bronchoscopy. Jung et al (26) indicate that 3DCTVB is a very safe and useful diagnostic technique for bronchial foreign bodies, and that 3D-CTVB should be used to diagnose all patients with suspected bronchial foreign bodies. According to Srivastava and Tyagi (28), VB is very useful in patients with compromised respiratory tract. VB is indicated as appropriate in planning a management protocol not only in relation to other conditions, but also on its own, but with accuracy below $100 \%$ due to false positive and false negative results. According to them, VB will not replace real bronchoscopy ("gold standard"), but it could help and supplement it. Yajima et al (31) mention VB as a method of non-invasive imaging and a potential technique for detecting a foreign body in the respiratory system, helping to determine the presence and exact location of the foreign body. Yogi et al (29) define VB as a new, relatively noninvasive procedure that provides a 3dimensional view of the inner walls of the tracheobronchial tree by reconstructing axial 
images. The authors state that VB should be performed after normal results of X-ray in patients with suspected foreign body to avoid unnecessary RB. They define VB as useful in screening the suggestions of a foreign body because of its specificity, sensitivity and validity.

The sensitivity, specificity and accuracy of thin-profile axial MDCT and MPR imaging in the diagnosis of aspirated foreign bodies are determined by Kocaoglu et al (5) respectively as $88.9 \%, 91.7 \%, 90.5 \%$. They obtain the same results as when VB is included. Bhat et al (2) (sensitivity $92.3 \%$, specificity, $85.7 \%$, validity $90 \%$, positive probability coefficient 6.45 and negative probability coefficient 0.089 ), Yogi et al (29) (sensitivity and specificity respectively 96.5 and $75 \%$, false positive $5.40 \%$ and false negative $2.70 \%$ ) etc. In the presence of positive clinical diagnosis and negative chest $\mathrm{X}$-ray, the authors recommend that VB with CT should be considered in all cases of aspiration of a tracheobronchial foreign body to avoid unnecessary RB. CT VB is determined as particularly useful in screening the cases of foreign body aspiration as it has a high sensitivity, specificity and validity.

\section{CONSLUSION}

MDST VB is a particularly useful modality in diagnosing and determining the exact location of cases of aspiration of a foreign body due to its high sensitivity, specificity and validity. Real bronchoscopy is a "gold standard". VB could help and supplement it and in children and adults and could avoid RB. VB is a method for non-invasive imaging and a potential technique for detecting foreign bodies in the respiratory system. VB does not allow, in case of inflammatory changes and the presence of secretions, to clearly define the form and type of the pathological finding. The preferred method used to remove foreign bodies is the FB.

\section{REFERENCE}

1. Haliloglu M, Ciftci AO, Oto A, Gumus B, Tanyel FC, Senocak ME, Buyukpamukcu $\mathrm{N}$, Besim A. CT virtual bronchoscopy in the evaluation of children with suspected foreign body aspiration. European $J$ of Radiology; $\quad 48(2): 188-192, \quad 2003$.
MITEV M. A., et al.

DOI: https://doi.org/10.1016/S0720048X(02)00295-4

2. Bhat KV, Hegde JS, Nagalotimath US and Patil GC. Evaluation of computed tomography virtual bronchoscopy in paediatric tracheobronchial foreign body aspiration. The Journal of Laryngology \& Otology; 124(8):875-879, 2010. https://doi.org/10.1017/S0022215110000769

3. Adaletli I, Kurugoglu S, Ulus S, Ozer H, Elicevik M, Kantarci F, Mihmanli I, Akman C. Utilization of low-dose multidetector CT and virtual bronchoscopy in children with suspected foreign body aspiration. Pediatric Radiology; $\quad 37(1): 33-40, \quad 2007$. http://dx.doi.org/10.1007/s00247-006-0331-y

4. James ET, Sun J, Ooi GC, Tsang KW. Endobronchial Foreign Body Presenting as Exacerbation of Asthma. Case Reports in Emergency Medicine; 1-5, 2017. https://doi.org/10.1155/2017/6863083

5. Kocaoglu M, Bulakbasi N, Soylu K, Demirbag S, Tayfun C \& Somuncu I. ThinSection Axial Multidetector Computed Tomography and Multiplanar Reformatted Imaging of Children with Suspected Foreign-Body Aspiration: Is Virtual Bronchoscopy Overemphasized? Acta Radiologica; 47(7):746-751, 2006.

6. Hosseinpour M, Kolahdouzan M. Needle in the Lung: A Difficult Case of a Pulmonary Foreign Body. Trauma Mon; 22(4):e13575, 2017. doi: 10.5812/traumamon.13575.

7. Laya BF, Restrepo R, Lee EY. Practical Imaging Evaluation of Foreign Bodies in Children: An Update. Radiol Clin N Am; 55: $\quad 845-867, \quad 2017$. http://dx.doi.org/10.1016/j.rcl.2017.02.012

8. Tsang JE, Sun J, Ooi GC, Tsang KW. Endobronchial Foreign Body Presenting as Exacerbation of Asthma. Case Reports in Emergency Medicine; 5 pp, 2017. https://doi.org/10.1155/2017/6863083

9. Simoff M, Bedi H. Foreign Bodies in the Airway: Endoscopic Methods. Interventions in Pulmonary Medicine; 547569, 2018. https://doi.org/10.1007/978-3319-58036-4_36

10.Jackson C. Bronchoscopy: past, present, and future. N Engl J Med; 199:758-63, 1928.

11.Prakash UBS. Bronchoscopy and Interventional Pulmonology. In: DíazJimenez J., Rodriguez A. (eds) Interventions in Pulmonary Medicine; Springer, Cham, 2018. https://doi.org/10.1007/978-3-319-580364_40

12.Hewlett JC, Rickman1 OB, Lentz RJ, Prakash UB, Maldonado F. Foreign body 
aspiration in adult airways: therapeutic approach. J Thorac Dis; 9(9):3398-3409, 2017.

13.Sersar SI, Rizk WH, Bilal M, El Diasty MM, Eltantawy TA, Abdelhakam BB, Elgamal AMF, Bieh AAA. Inhaled Foreign Bodies: Presentation, Management and Value of History and Plain Chest Radiography in Delayed Presentation. Otolaryngology-Head and Neck Surgery; 134, 92-99, 2006. doi:10.1016/j.otohns.2005.08.019

14.Codeso FMP, Galindo AD, Fernández DJ, Varela MA, Rueda MG, de la Cruz Ríos JL. Descriptive Anal ysis of 10 Years of Interventional Therapeutic Bronc hoscopy. Rev Esp Patol Torac; 29(2):125-133, 2017.

15.Digra SK, Kishore K, Digra KK, Slathia SS. Ear and aerodigestive tract foreign body in children in a tertiary care centre in North India. J. Evolution Med. Dent Sci; 6(87):5997-6000, $2017 . \quad$ DOI: $10.14260 /$ jemds/2017/1304

16. Flandes J, Cascon JA, Cordovilla R, Alfayate J. Foreign Body Retrieval. In: Frontiers in Respiratory Medicine. Advances in Interventional Pulmology (Ali I Musani, Ed); 1(20):356-372, 2017. ISSN(Online): 2589-1855

17. Tauseef U, Agha F, Ehsan S, Bukhari SI, Tauseef A, Tauseef A. Subcutaneous emphysema: A rare presentation of bronchial foreign body overlooked by Paediatricians. International Journal of Research; 4(7): 1096-1099, 2017. https://edupediapublications.org/journals/in dex.php/IJR/

18.Dabu J, Lindner M, Azzam M, Al-Khateeb A, Kadri M, Bellary S, Miller R. A Case of Chronic Cough and Pneumonia Secondary to a Foreign Body. Case Reports in Medicine; $\quad 1-3, \quad 2017$. https://doi.org/10.1155/2017/3092623

19.Eyekpegha OJ, Onakpoya UU, Obiajunwa PO, Famurewa OC, Ogunrombi AB. Missed distal tracheal foreign body in consecutive bronchoscopies in a 6-year-old boy. Niger J Surg; 23:67-70, 2017. DOI: 10.4103/1117-6806.199957

20.Shashidhar K, Rajan RRV, Shah N, Bhat N. Rigid bronchoscopy for foreign body removal: an overview. International Journal of Otorhinolaryngology and Head and Neck Surgery; 3(4):936-938, 2017. DOI: http://dx.doi.org/10.18203/issn.24545929.ijohns20173170

21.Wani NA, Qureshi UA, Kosar T, Iaway MA. Subcutaneous emphysema due to bronchial foreign body demonstrated by multidetector-row computed tomography.
MITEV M. A., et al. Lung India; 28(4):291-293, 2011. doi: $10.4103 / 0970-2113.85693$

22.Kulkarni G, Ambadekar S. Study of Clinicoradiological Profile of Patients Undergoing Fiberoptic Bronchoscopy. MVP Journal of Medical Sciences; 4(1):6469, 2017. https://doi.org/10.18311/mvpjms/2017/v4i1 $/ 15609$

23.Sattar A, Ahmad I, Javed AM, Anjum S. Diagnostic accuracy of chest $\mathrm{x}$-ray in tracheobronchial foreign body aspiration in paediatric patients. J Ayub Med Coll Abbottabad; 23(4):103-105, 2011.

24.Koşucu P, Ahmetoğlu A, Koramaz I, Orhan F, Özdemir O, Dinç H, Ökten A, Gümele HR. Low-Dose MDCT and Virtual Bronchoscopy in Pediatric Patients with Foreign Body Aspiration. AJR; 183:17711777, 2004.

25.Cevizci N, Dokucu Al, Baskin D, Karadağ CA, Sever N, Yalçin M, et al. Virtual bronchoscopy as a dynamic modality in the diagnosis and treatment of suspected forein body aspiration. Eur J Pediatr Surg; 18(6):398-401, 2008. DOI: 10.1055/s-20081038972

26.Jung S, Pae S, Chung S, Kim H. Threedimensional CT with virtual bronchoscopy: a useful modality for bronchial foreign bodies in pediatric patients. European Archives of Oto-Rhino-Laryngology; 269(1):223-228, 2012. DOI: 10.1007/s00405-011-1567-1

27.Richards AM. Pediatric Respiratory Emergencies. Emergency Medicine Clinics; 34(1):77-96, 2016. DOI: https://doi.org/10.1016/j.emc.2015.08.006

28.Srivastava M, Tyagi S. Usefulness of virtual bronchoscopy in evaluation of suspected foreign body in tracheobronchial tree. Int J Otorhinolaryngol Head Neck Surg; 2(1):18-21, 2006.

29.Jogi M, Surender K, Jadi L, Chavan S, Pratihiba P. Advantage of Computed Tomography (CT) Virtual Bronchoscopy in the Evaluation of Children with Suspected Foreign Body Airway. Int J Otolaryngol Head Neck Surg; 5:59-64, 2016.

30.Hou R, Zhou H, Hu K, Ding Y, Yang $\mathrm{X}, \mathrm{Xu}$ G, Xue P, Shan C, Jia S, Ma Y. Thorough documentation of the accidental aspiration and ingestion of foreign objects during dental procedure is necessary: review and analysis of 617 cases. Head \& Face Medicine; 12:23, 2016. DOI 10.1186/s13005-016-0120-2

31. Yajima H, Tomita K, Fujii Y, Ueda Y, Shimizu E. Bronchial Foreign Body Attributable to an Aspirated Marking Pin: 
A Case Report. J Pulm Respir Med; 6 (2):x324,

2016.

http://dx.doi.org/10.4172/2161-

105X.1000324

32.Elhamady HAE, Ramadan AEM, Gaafar AH, Baess AI, Hammad SW. Incidence, patterns and different modalities in extraction of aero-digestive tract foreign bodies in patients attending Alexandria Main University Hospital. Journal of the Egyptian Society of Cardio-Thoracic Surgery; 25(2):154-162, 2017. https://doi.org/10.1016/j.jescts.2017.05.002

33. Gomes SBE, Alves G, Marchiori E, Hochhegger B. Chest CT findings in patients with dysphagia and aspiration: a systematic review. J. Bras Pneumol; 43(4), $2017 . \quad$ http://dx.doi.org/10.1590/s180637562016000000273

34.Mitev MA. Virtual bronchoscopy with Multidetector computer tomography. $\mathrm{PhD}$ These. Trakia University, Stara Zagora, 180, 2017.

35.Mitev MA. Virtual bronchoscopy with Multidetector computer tomography. Autoreferate of $\mathrm{PhD}$ These. Trakia University, Stara Zagora, 40, 2017.

36. Mitev MA, Trajkova N, Arabadzhiev D, Valkanov S, Georgieva N, Obretenov E. Multidetector computed tomography approach to the diagnosis of a foreign body. Trakia J Sci; 15(3):198-202, 2017. ISSN 1313-7050

37. Kostadinov D. Foreign bodies in the trachea and bronchus. J MedInfo; 2011.

38. Boninska N, Borisova I. Imaging methods for detecting of foreign body in respiratory system. J Med forum; 3(4):71-74, 2014.

39. Pitiot V, Grall M, Ploin D, Truy
MITEV M. A., et al. E, Khalfallah SA, Metrics PX. The use of $\mathrm{CT}$-scan in foreign body aspiration in children: A 6 years' experience. International J. of Pediatric Otorhinolaryngology; 102: 169-173, 2017. https://doi.org/10.1016/j.ijporl.2017.08.036

40. Reynisson PJ, Hofstad EF, Leira CA, Langø Th, Sorger H. A new visualization method for navigated bronchoscopy. Journal Minimally Invasive Therapy \& Allied Technologies; 1-8, 2017. https://doi.org/10.1080/13645706.2017.132 7870

41. Semple T, Calder A, Owens CM, Padley S, Metrics PX. Current and future approaches to large airways imaging in adults and children. Clinical Radiology; 72(5):356374, 2017. https://doi.org/10.1016/j.crad.2017.01.012

42. Tartar T, Bakal U, Onur MR, Saraç M, Poyraz AK, Ahmet K. Assessment of Foreign Body Aspiration Material Using a Virtual Bronchoscopy Model: TimeDensity and Time-Volume Relationships. Iran J Radiol; 14(2):e42990, 2017. doi: 10.5812/iranjradiol.42990

43. Konen E, Katz M, Rozenman J, Ben-Shlush A, ltzchak Y, Szeinberg A. Virtual Bronchoscopy in Children: EarlyClinicalExperience. AJR; 171:16991702, 1998. www.ajronline.org by 194.141.53.208

44. Nakagava BT, Penteado RVS, Tsuzuki MSG, Tori R, Takimoto RY, Martins TC, Abe LI, Rosso Jr RSU, Gotoh T, Iwasawa T. Virtual Reality of Animated Lung with Internal Structures. IFAC PapersOnLine, 50-1: $\quad$ 15151-15156, 2017. https://doi.org/10.1016/j.ifacol.2017.08.2258 